\title{
Desempenho, composição do leite e metabólitos sanguíneos de vacas Holandês x Gir manejadas em pastagem de Brachiaria brizantha cv. Marandu e suplementadas com grão de soja tostado
}

[Performance milk composition and blood metabolites of Holstein x Gir cows grazing Brachiaria brizantha $c v$. Marandu grass supplemented with roasted soybeans]

\author{
M.H.F. Mourthe ${ }^{1}$, R.B. Reis $^{2}$, F.C.F. Lopes $^{3,4}$, M.A.S. Gama ${ }^{3}$, R.C.Souza ${ }^{1}$ \\ ${ }^{1}$ Médico veterinário autônomo - Belo Horizonte, MG \\ ${ }^{2}$ Escola de Veterinária - UFMG - Belo Horizonte, MG \\ ${ }^{3}$ Embrapa Gado de Leite - Juiz de Fora, MG \\ ${ }^{4}$ Bolsista de produtividade do $\mathrm{CNPq}$
}

\begin{abstract}
RESUMO
Avaliaram-se o consumo, metabólitos sanguíneos e a produção e composição do leite de 16 vacas Holandês x Gir, manejadas em pastagem de Brachiaria brizantha cv. Marandu, suplementadas com $6 \mathrm{~kg} / \mathrm{vaca} / \mathrm{dia}$ (base matéria natural) de concentrado contendo $0 ; 1,3 ; 2,6$ e 3,9kg/vaca/dia de grão de soja tostado (GST). Foi utilizado o delineamento com quatro quadrados latinos (QL) 4 x 4, sendo cada fase do QL constituída de 10 dias de período de adaptação à dieta e de cinco para coleta de amostras. A suplementação da dieta com GST resultou em redução linear $(\mathrm{P}<0,05)$ nos consumos de matéria seca e de fibra insolúvel em detergente neutro do pasto e total. A concentração de glicose não foi afetada $(\mathrm{P}>0,05)$, porém a de ácidos graxos não esterificados aumentou com a inclusão do GST na dieta $(\mathrm{P}<0,05)$. Não houve efeito $(\mathrm{P}>0,05)$ da adição do GST sobre a produção e composição do leite, exceto para o teor $(\mathrm{P}=0,10)$ e produção $(\mathrm{P}=0,08)$ de gordura no leite.
\end{abstract}

Palavras-chave: bovino, gramínea tropical, lipídeos, processamento, suplementação concentrada

\begin{abstract}
Feed intake, blood metabolites, and milk yield and composition of Holstein x Gir cows grazing Brachiaria brizantha $c v$. Marandu receiving $6 \mathrm{~kg} / \mathrm{cow} /$ day (natural matter based) of concentrate containing 0, 1.3, $2.6,3.9 \mathrm{~kg} / \mathrm{cow} /$ day of roasted soybean seeds (RSS) were evaluated. The experimental design was four $4 x$ 4 latin square $(L S)$ in wich each phase consisted of 10 days for diet adaptation period and 5 days for samples collection. Diet supplementation with RSS decreased linearly $(P<0.05)$ the intake of pasture, total diet dry matter and neutral detergent fiber. The plasma glucose concentration was unaffected $(P>0.05)$, but nonesterified fatty acids increased with RSS supplementation $(P<0.05)$. There was no effect $(P>0.05)$ of RSS supplement on milk production and composition, except the linear reduction on the content $(P=0.10)$ and yield $(P=0.08)$ milk fat, which can present positive financial implication to farmers, knowing that most of the Brazilian dairy use payment programs by milk composition.
\end{abstract}

Keywords: cattle, tropical grass, lipids, processing, concentrate supplement

\section{INTRODUÇÃO}

$\mathrm{Na}$ produção intensiva de leite em pastagem, vacas de maior produção normalmente necessitam da suplementação concentrada para complementarem os nutrientes não fornecidos pelos pastos. Os suplementos concentrados que utilizam o farelo de soja e o milho possuem degradação ruminal mais rápida que a da fibra do pasto. Mesmo que fracionada a suplementação concentrada pode representar elevada ingestão de carboidrato/proteína rapidamente degradáveis no

Recebido em 19 de julho de 2011

Aceito em 12 de janeiro de 2012

E-mail: kikolider@yahoo.com.br 
rúmen, implicando em eventuais distúrbios digestivos/metabólicos, caso haja acúmulo de amônia e ácidos, pela insuficiente utilização/remoção do rúmen (Pereira e Armentano, 2000). Além disso, o fornecimento de quantidades elevadas de concentrados ricos em carboidratos não estruturais pode provocar queda no teor de gordura do leite, depressão na degradabilidade da fibra, acidose e redução do consumo de matéria seca (MS). A inclusão de lipídeos insolúveis na dieta pode evitar estes distúrbios (Van Soest, 1994).

A soja tostada pode ajudar neste equilíbrio ao diminuir a degradação ruminal da proteína e liberar o óleo lentamente, minimizando os efeitos deletérios deste aos microrganismos do rúmen (Dhiman et al., 1997). Além disso, o aporte desses nutrientes pode aumentar a digestibilidade total da dieta e, consequentemente, o consumo de MS, com reflexos positivos sobre o desempenho animal.

O processamento térmico da soja também apresenta a vantagem de eliminar fatores anti-nutricionais, que diminuem a digestibilidade dos nutrientes (Silva et al., 2002), reduzir a fração de proteína degradável no rúmen (PDR), além de disponibilizar o óleo lentamente dentro do rúmen. A soja processada termicamente constitui-se na melhor fonte vegetal de proteína não degradável no rúmen (PNDR), pois foi a que mais aproximou do perfil de aminoácidos da proteína microbiana, apesar de sua deficiência em metionina (Santos et al., 1998).

Além disso, o óleo do grão de soja é rico em ácidos graxos poli-insaturados (AGPI), principalmente o ácido linoleico (C18:2 cis-9,cis-12), importante precursor do ácido rumênico (C18:2 cis-9,trans-11), um isômero conjugado do ácido linoleico, e ao qual foram atribuídas características nutracêuticas, devido as propriedades anti-cancerígenas e antitrombogênicas (Tricon e Yaqoob, 2006). O óleo da soja apresenta potencial de aumentar os teores de ácido rumênico no leite de vacas recebendo forrageira tropical como base da dieta (Lopes et al., 2009).
Entretanto, o estudo da suplementação com grão de soja tostada (GST) sobre a ingestão e desempenho de vacas lactantes sob pastejo de forrageiras de clima tropical faz-se necessário, pois tem potencial de substituir o farelo de soja, com a vantagem de agregar maior valor ao leite.

O objetivo deste estudo foi avaliar a inclusão de quantidades crescentes de GST no concentrado de vacas em lactação manejadas em pastagem de Brachiaria brizantha cv. Marandu sobre o consumo de matéria seca, metabólitos sanguíneos, produção e composição do leite.

\section{MATERIAL E MÉTODOS}

O experimento foi realizado no período de 6 de fevereiro a 10 de abril de 2009. Os procedimentos experimentais utilizados foram aprovados pelo Comitê de Ética em Experimentação Animal da Universidade Federal de Minas Gerais (Protocolo CETEA 196/08). Utilizaram-se 16 vacas Holandês $\mathrm{x}$ Gir, multíparas, no terço inicial de lactação $(58 \pm 20$ dias), produzindo $20 \pm 5 \mathrm{~kg}$ de leite/dia, e pesando $506 \pm 51 \mathrm{~kg}$, em pastagem de Brachiaria brizantha cv. Marandu. Foi utilizado delineamento com quatro quadrados latinos (QL) 4 x 4 contemporâneos, sendo cada fase do QL constituída por 10 dias de período de adaptação à dieta e de cinco para coletas de amostras. Os tratamentos experimentais foram: T0 - suplementação concentrada sem a inclusão de grão de soja tostado (GST); T1,3 - suplementação concentrada contendo 1,3kg/vaca/dia de GST; T2,6 - suplementação concentrada contendo 2,6kg/vaca/dia de GST e T3,9 - suplementação concentrada contendo 3,9kg/vaca/dia de GST (Tab. 1).

Os animais receberam $6 \mathrm{~kg} / \mathrm{dia}$ (base matéria natural) dos suplementos concentrados, em cochos eletrônicos individuais do tipo calan-gate (American Calan Inc., Northewwod, NH, EUA), fracionados em duas vezes ao dia após as ordenhas da manhã e da tarde, que foram iniciadas às 6 e 14h, respectivamente. 
Tabela 1. Ingredientes (kg/dia) e composição química (\% da matéria seca) dos concentrados contendo quantidades crescentes de grão de soja tostado

\begin{tabular}{lcccc}
\hline \multirow{2}{*}{ Ingrediente (kg/dia) } & \multicolumn{4}{c}{ Concentrado } \\
\cline { 2 - 4 } & T 0 & T 1,3 & T 2,6 & T 3,9 \\
\hline Grãos de soja tostada (GST) & 0 & 1,3 & 2,6 & 3,9 \\
Farelo de soja & 2,7 & 1,9 & 1,2 & 0,5 \\
Milho moído & 1,6 & 1,3 & 1,0 & 0,7 \\
Polpa cítrica & 1,3 & 1,3 & 1,0 & 0,7 \\
MMV & 0,2 & 0,2 & 0,2 & 0,2 \\
Composição química (\% da MS) & & & \\
Matéria seca (MS) & 84,4 & 85,3 & 84,8 & 86,1 \\
Proteína bruta (PB) & 28,5 & 30,4 & 30,9 & 32,9 \\
Fibra em detergente neutro (FDN) & 16,8 & 17,4 & 21,0 & 20,1 \\
Fibra em detergente ácido (FDA) & 9,1 & 9,3 & 11,1 & 10,8 \\
Extrato etéreo (EE) & 2,6 & 8,7 & 11,3 & 16,5 \\
Carboidratos não fibrosos (CNF) & 43,1 & 35,4 & 28,5 & 22,9 \\
Cinzas & 9,0 & 8,1 & 8,3 & 7,6 \\
\hline
\end{tabular}

Tratamentos: T0; T1,3; T2,6 e T3,9, respectivamente, suplemento concentrado sem e com a inclusão de 1,3; 2,6 e 3,9kg/vaca/dia de GST; MMV = mistura mineral vitamínica; PB- proteína bruta; FDN - fibra insolúvel em detergente neutro; FDA - fibra insolúvel em detergente ácido; EE - extrato etéreo; $\mathrm{CNF}$ = carboidratos não fibrosos.

O GST foi quebrado em moinho dotado de peneira com abertura de malha de $10 \mathrm{~mm}$, antes de ser misturado aos suplementos. A tostagem do grão foi realizada à temperatura de $145^{\circ}$ a $149^{\circ} \mathrm{C}$ por 30 a 45 segundos, com $35 \mathrm{~min}$ de steeping (armazenamento em recipiente térmico após o processamento, para que o calor penetre mais profundamente dentro do grão).

Todas as vacas foram mantidas na mesma pastagem de 9,5ha de $B$. brizantha com disponibilidade mínima de $153 \mathrm{~kg}$ de $\mathrm{MS}$ por vaca por dia. No primeiro dia do período de adaptação de cada fase do QL, foi colhida forragem selecionada na pastagem, utilizando-se uma vaca em lactação fistulada no esôfago, dotada de bolsa para coleta de extrusas, adaptada ao pastejo. A duração da coleta foi de, aproximadamente, 40min, após a primeira ordenha.

As amostras de extrusa foram acondicionadas em sacos plásticos para o transporte até o laboratório, e armazenadas $\left(-10^{\circ} \mathrm{C}\right)$. Posteriormente, foram descongeladas, compostas de acordo com a fase experimental do QL, présecadas em estufa de circulação forçada de ar $\left(55^{\circ} \mathrm{C}, 72 \mathrm{~h}\right)$, sendo, posteriormente, moídas (1mm) e analisadas (Silva e Queiroz, 2002) quanto aos teores de matéria seca $(\mathrm{MS})$ a $105^{\circ} \mathrm{C}$, de proteína bruta $(\mathrm{PB})$, de extrato etéreo $(\mathrm{EE})$, de fibra insolúvel em detergente neutro (FDN) e em detergente ácido (FDA) e lignina. Parte dessas amostras foi utilizada para o ensaio de digestibilidade in vitro de MS (DIVMS), conforme método descrito por Tilley e Terry (1963).

Foram realizadas amostragens diárias dos suplementos concentrados e dos ingredientes utilizados, sendo estas transformadas, posteriormente, em compostas representativas de cada suplemento em cada fase do QL e analisadas conforme relatado para as extrusas. Amostras de líquido ruminal de quatro vacas fistuladas no rúmen pastejando no mesmo piquete e recebendo as mesmas dietas deste experimento foram coletadas para determinação da DIVMS, realizada na Incubadora In vitro modelo TE-150 (Tecnal Equipamentos para Laboratório, Piracicaba, SP), utilizando filter bags F57 (Ankom Technology, Macedon, NY, EUA).

A amostragem do pasto para determinação da massa de forragem disponível na pastagem foi feita no primeiro dia de cada fase do QL, com auxílio de um quadrado $(0,5 \times 0,5 \mathrm{~m})$ e com cortes realizados a $5 \mathrm{~cm}$ do nível do solo, lançados em 30 áreas escolhidas ao acaso dentro do piquete (adaptado de McMeniman, 1997). Após a pesagem das 30 amostras, separou-se o material 
senescente ("matéria morta") e o verde restante foi separado em folhas e caule. Todas as partes fracionadas foram pesadas para posterior caracterização da relação folha:caule, présecadas $\left(55^{\circ} \mathrm{C}, 72 \mathrm{~h}\right)$ e, posteriormente, moídas $(1 \mathrm{~mm})$ e analisadas quanto à composição química, conforme descrito para a extrusa.

O consumo individual diário de MS foi estimado em cada fase do QL nas 16 vacas, a partir da fórmula: Consumo (kg de MS) = Produção fecal x 100/(100 - DIVMS). Para a estimação da produção fecal total foi utilizado o óxido crômico $\left(\mathrm{Cr}_{2} \mathrm{O}_{3}\right)$ como indicador externo, na quantidade de $10 \mathrm{~g} / \mathrm{vaca} / \mathrm{dia}$, divididos em duas partes iguais e embalados em papel-toalha, sendo administrado por via oral às vacas do quarto ao $15^{\circ}$ dia de cada fase do QL, sempre após as duas ordenhas. As coletas de fezes foram realizadas duas vezes ao longo dos últimos cinco dias, sempre após os horários das ordenhas, e armazenadas $\left(-10^{\circ} \mathrm{C}\right)$. Posteriormente, foram descongeladas, pré-secadas em estufa de ventilação forçada $\left(55^{\circ} \mathrm{C}, 72 \mathrm{~h}\right)$, moídas $(1 \mathrm{~mm})$ e compostas com base no peso seco, por vaca e por fase do QL. As amostras foram analisadas quanto ao teor de cromo, por espectrofotometria de absorção atômica (Williams et al., 1962), após digestão nitroperclórica (Kimura e Miller, 1952). A produção fecal relativa ao consumo de pasto foi calculada por diferença entre a total e aquela referente ao consumo dos suplementos concentrados, conforme realizado por Lopes et al. (2004).

No período de coleta, amostras de leite individuais em duplicata, referentes a cinco ordenhas consecutivas, foram coletadas e acondicionadas em frascos contendo conservante bronopol ${ }^{\circledR}$ para posterior determinação dos teores de proteína, gordura, lactose, extrato seco total e nitrogênio ureico do leite (NUL), segundo procedimentos da AOAC (Official..., 1990). As análises de composição do leite foram realizadas em equipamento Bentley ${ }^{\circledR} 2000$ (Bentley Instruments Inc., Chaska, Minnesota, EUA) no Laboratório de Qualidade de Leite da Embrapa Gado de Leite em Juiz de Fora, MG, sendo a análise do NUL realizada na Clínica do Leite da ESALQ/USP em Piracicaba, SP.

No $15^{\circ}$ dia de cada fase experimental, imediatamente após a ordenha, foi realizada coleta de amostras de sangue de cada vaca, por meio de punção dos vasos coccígenos. As amostras foram então, armazenadas em tubos contendo heparina (Thompson e Christie, 1991), e centrifugadas a $3.200 \mathrm{~g}$ por $15 \mathrm{~min}$. O plasma obtido foi pipetado, transferido para tubos e armazenado a $-10^{\circ} \mathrm{C}$ (Wonsil et al., 1994), até a realização das análises das concentrações de glicose utilizando-se o analisador automático "Biochemistry Analyser Model 2700 Select" (YSI Inc., Yellow Springs, Ohio, EUA). Outra amostra de sangue foi coletada da mesma maneira anterior, porém utilizou-se tubos sem anti-coagulante, para análise de ácidos graxos não esterificados (NEFA) por meio de kit comercial (NEFA C, Wako Pure Chemicals Industries Ltd., Osaka, Japão).

Os resultados foram analisados pelo procedimento GLM do SAS (2002). Os efeitos das quantidades de inclusão de GST no concentrado foram avaliados por meio de análises de regressão linear e quadrática pelo PROC REG do SAS (2002).

\section{RESULTADOS E DISCUSSÃO}

A massa de forragem disponível durante todo o período experimental sempre foi superior a $4.823 \mathrm{~kg}$ de $\mathrm{MS} / \mathrm{ha}$, ou seja, maior que os $2.000 \mathrm{~kg}$ de MS/ha, considerados por Minson (1990) como a quantidade mínima necessária para não interferir no consumo e, consequentemente, no desempenho dos animais. A relação folha:caule:matéria morta média foi de 32:51:17, refletindo o manejo de pastejo contínuo da pastagem. Considerando apenas a relação folha:caule, a média foi de 35:65. A taxa de lotação média do piquete foi de 2,4 UA/ha e não variou durante os 60 dias experimentais.

A composição química da extrusa foi de 12,5; 8,$9 ; 10,8 ; 1,2 ; 69,7 ; 35$ e $5 \%$ de MS, cinzas, PB, EE, FDN, FDA e lignina, na base da MS, respectivamente. Lopes et al. (2010) relataram que a forragem selecionada em piquete de capim-brachiária, obtida da amostra de extrusa coletada de vaca em lactação fistulada no esôfago apresentou 12,2\% de MS, 10,2\% de PB e $66,8 \%$ de FDN, valores similares aos obtidos no presente experimento. A amostra de pasto colhida por meio de corte manual apresentou 34,$4 ; 8,4 ; 4,1 ; 1,5 ; 74,9 ; 40,9$ e $5,2 \%$ de MS, cinzas, PB, EE, FDN, FDA e lignina, na base da MS, respectivamente. Estes valores refletem a 
seleção feita pelas vacas sob pastejo, principalmente em relação aos teores de PB. Foi observado que a extrusa era constituída, em sua maior parte, de folhas verdes, porém também apresentava pequenas hastes e folhas secas, o que pode explicar o valor encontrado para lignina.

Houve redução linear $(\mathrm{P}<0,05)$ na DIVMS do concentrado, porém não houve diferença para a extrusa $(\mathrm{P}>0,05)$, com o aumento da participação do GST no suplemento concentrado (Tab. 2). O valor médio encontrado para a DIVMS da extrusa foi de $66,6 \%$, semelhante aos relatados por Valadares Filho et al. (2010) de 68,9 e $65,5 \%$ para amostras de B. brizantha cv. Marandu de 16 a 30 e de 31 a 45 dias de crescimento, respectivamente.

O efeito encontrado para a DIVMS dos concentrados reflete o processamento e o aumento do GST nos concentrados. Em função da adição de GST houve redução dos teores de milho e polpa cítrica, ricos em carboidratos não fibrosos digestíveis no rúmen, além da menor digestibilidade da fração proteica no rúmen. A redução linear da DIVMS do suplemento concentrado T0 para T3,9 foi de $11,3 \%$.

Tabela 2. Digestibilidades in vitro da matéria seca (\%) da extrusa de Brachiaria brizantha cv. Marandu e dos concentrados experimentais

\begin{tabular}{lcccccc}
\hline \multirow{2}{*}{ Alimento } & \multicolumn{4}{c}{ Concentrado } & \multirow{2}{*}{ EPM } & \multirow{2}{*}{ Equação de regressão } \\
\cline { 2 - 4 } & T0 & T1,3 & T2,6 & T3,9 & & \\
\hline Concentrado, \% & 95,4 & 94,1 & 90,7 & 84,6 & 1,195 & $\hat{y}=96,57-2,38 X\left(\mathrm{r}^{2}=0,75\right)$ \\
Extrusa, \% & 65,7 & 67,5 & 68,4 & 64,8 & 1,796 & ns \\
\hline
\end{tabular}

Concentrados: T0; T1,3; T2,6 e T3,9 - suplementos concentrados sem inclusão de grão de soja tostado (GST) e com inclusão de 1,3; 2,6 e 3,9kg de GST (base matéria natural); EPM = Erro-padrão da média ; ns = não-significativo $(\mathrm{P}>0,05)$

Houve redução linear $(\mathrm{P}<0,05)$ da quantidade crescente de GST sobre o consumo de MS e de FDN total, e de MS de pasto (kg/vaca/dia e porcentagem do peso corpóreo -\%PV) (Tab. 3). $\mathrm{Na}$ formulação das dietas experimentais, realizada com o software Spartan (Michigan State University) o consumo de MS total e de forragem foi projetado ser em torno de 15 e $9 \mathrm{~kg}$ de MS/dia, respectivamente. De certa forma, demonstrou-se que o uso de óxido crômico como indicador, estimou bem a produção fecal e, consequentemente, o consumo de pasto.

A literatura demonstrou efeito do grão de soja "in natura" ou tostado na redução do consumo de MS (4 a 27\%) em experimentos com diferentes inclusões do grão (13 a 27\% na MS) tanto na dieta de bovinos como de outras espécies (Vargas et al., 2002; Jordan et al., 2006; Oliveira et al., 2007; Silva et al., 2007; Souza et al., 2009). Exceto no trabalho de Silva et al. (2007), em todos os demais, silagens de milho ou sorgo foram a base das dietas, demonstrando a relevância do presente estudo em fornecer informações sobre a suplementação de lipídeos por meio do GST para vacas em lactação manejadas em pastagem de clima tropical. Os autores relataram pouco efeito do óleo diretamente sobre microrganismos do rúmen, atribuindo a redução do consumo ao efeito de saciedade metabólica provocados pelos AGPI (Allen, 2000) e, provavelmente, este mesmo efeito pode ter ocorrido no presente estudo devido ao GST, pois as vacas que receberam o T3,9 apresentaram consumo de MS e FDN, 11,6 e $14,6 \%$, respectivamente, menores que as vacas que receberam $\mathrm{T} 0$.

$\mathrm{O}$ consumo quando expresso em \%PV apresentou variações de 3,0 a 2,5; 2,0 a 1,5 e de 1,6 a $1,2 \%$ para MS total, MS do pasto e FDN total, respectivamente (Tab. 3). Revisões de literatura com dados de vacas manejadas em pastagens formadas com forrageiras de clima tropical suplementadas com diferentes quantidades de concentrado relataram média de consumo de $2,35 \pm 0,61 \% \mathrm{PV}$ para $\mathrm{MS}$, $1,67 \pm 0,48 \%$ PV para FDN (Reis e Souza, 2007) e de 2,61 a 3,53\%PV e 1,65 a 2,57\%PV, respectivamente, para o consumo de MS total e de MS do pasto (Lopes, 2008). Portanto, mesmo com o efeito da suplementação do GST, os consumos no presente estudo foram semelhantes aos observados na literatura. Ressalta-se que mesmo com o alto teor de FDN da forragem $(69,7 \%)$, a suplementação concentrada correspondeu à, aproximadamente, $40 \%$ da dieta em todos os tratamentos, o que pode ter contribuído para os valores encontrados. 
Tabela 3. Consumos diários (kg/vaca ou \% do peso vivo - \%PV) de matéria seca (MS), fibra insolúvel em detergente neutro (FDN), proteína bruta (PB), carboidratos não fibrosos (CNF), extrato etéreo (EE) e ácidos graxos $\alpha$-linolênico (C18:3 cis-9 cis-12 cis-15), linoleico (C18:2 cis-9 cis-12) e oleico (C18:1 cis-9) de vacas em pastagem de Brachiaria brizantha cv. Marandu suplementada com 0; 1,3; 2,6 e $3,9 \mathrm{~kg} / \mathrm{vaca} / \mathrm{dia}$ de grão de soja tostado no concentrado

\begin{tabular}{|c|c|c|c|c|c|c|}
\hline \multirow{2}{*}{ Consumo } & \multicolumn{4}{|c|}{ Concentrado } & \multirow{2}{*}{ EPM } & \multirow{2}{*}{ Equação de regressão } \\
\hline & T0 & $\mathrm{T} 1,3$ & $\mathrm{~T} 2,6$ & T3,9 & & \\
\hline \multicolumn{7}{|l|}{ Consumo de MS } \\
\hline Total, kg & 15,4 & 15,0 & 14,6 & 12,8 & 0,238 & $\hat{y}=15,83-0,64 X\left(r^{2}=0,09\right)$ \\
\hline Total, \%PV & 3,02 & 2,98 & 2,83 & 2,50 & 0,144 & $\hat{\mathrm{y}}=3,11-0,14 \mathrm{X}\left(\mathrm{r}^{2}=0,09\right)$ \\
\hline Pasto, $\mathrm{kg}$ & 10,4 & 9,8 & 9,5 & 7,6 & 0,734 & $\hat{\mathrm{y}}=10,75-0,66 \mathrm{X}\left(\mathrm{r}^{2}=0,10\right)$ \\
\hline Pasto, \% PV & 2,03 & 1,96 & 1,84 & 1,49 & 0,144 & $\hat{\mathrm{y}}=2,11-0,14 \mathrm{X}\left(\mathrm{r}^{2}=0,10\right)$ \\
\hline \multicolumn{7}{|l|}{ Consumo de FDN } \\
\hline Total, kg & 8,08 & 7,76 & 7,71 & 6,37 & 0,511 & $\hat{y}=8,34-0,40 X\left(r^{2}=0,07\right)$ \\
\hline Total, \%PV & 1,58 & 1,55 & 1,49 & 1,24 & 0,100 & $\hat{y}=1,64-0,85 X\left(r^{2}=0,08\right)$ \\
\hline Pasto, kg & 7,23 & 6,86 & 6,64 & 5,34 & 0,511 & $\hat{\mathrm{y}}=7,49-0,46 \mathrm{X}\left(\mathrm{r}^{2}=0,10\right)$ \\
\hline Pasto, \%PV & 1,41 & 1,37 & 1,28 & 1,04 & 0,100 & $\hat{\mathrm{y}}=1,47-0,09 \mathrm{X}\left(\mathrm{r}^{2}=0,10\right)$ \\
\hline Consumo de PB, g & 2.563 & 2.628 & 2.601 & 2.526 & 79,23 & ns \\
\hline Consumo de $\mathrm{CNF}, \mathrm{g}$ & 3.159 & 2.746 & 2.348 & 1.902 & 68,88 & $\hat{y}=3.176-321\left(r^{2}=0,77\right)$ \\
\hline Consumo de EE, g & 256,1 & 565,1 & 690,1 & 943,3 & 9,035 & $\hat{y}=287+168\left(r^{2}=0,96\right)$ \\
\hline \multicolumn{7}{|l|}{ Consumo de $\mathrm{AG}$} \\
\hline Ácido $\alpha$-linolênico, g & 57,5 & 78,3 & 86,5 & 96,0 & 3,740 & $\hat{y}=61,7+9,47\left(r^{2}=0,48\right)$ \\
\hline Ácido linoleico, $g$ & 82,0 & 253,6 & 323,7 & 471,1 & 1,809 & $\hat{\mathrm{y}}=97,7+95,2\left(\mathrm{r}^{2}=0,98\right)$ \\
\hline Ácido oleico, g & 38,3 & 103,9 & 130,6 & 189,9 & 0,460 & $\hat{\mathrm{y}}=43,4+37,1\left(\mathrm{r}^{2}=0,98\right)$ \\
\hline
\end{tabular}

Concentrados: T0; T1,3; T2,6 e T3,9 - suplementos concentrados sem inclusão de grão de soja tostado (GST) e com inclusão de 1,3; 2,6 e 3,9kg de GST (base matéria natural); EPM = Erro-padrão da média ; ns = não-significativo $(\mathrm{P}>0,05) ; \mathrm{AG}=$ ácidos graxos.

O consumo diário de EE pelas vacas aumentou $(\mathrm{P}<0,05)$ com a inclusão crescente de GST no concentrado, devido ao maior teor de EE destes suplementos. Em relação ao consumo de $\mathrm{CNF}$ houve redução linear $(\mathrm{P}<0,05)$, com o aumento da suplementação de GST no concentrado. Estas respostas foram devidas a inclusão decrescente do milho moído e polpa cítrica para as dietas T0; T1,3; T2,6 e T3,9, já que esses ingredientes foram a principal fonte de amido e pectina, respectivamente, utilizada nos suplementos concentrados.

Os consumos dos ácidos graxos (AG) $\alpha$ linolênico (C18:3 cis-9,cis-12,cis-15), linoleico (C18:2 cis-9, cis-12) e oleico (C18:1 cis-9) aumentaram linearmente $(\mathrm{P}<0,05)$ com a inclusão crescente de GST no concentrado. Estes resultados podem ser atribuídos ao perfil de AG das dietas experimentais. Os principais AG do pasto e do GST foram o $\alpha$-linolênico ( $42 \%$ do AG totais) e linoleico (53\% dos AG totais), respectivamente. A redução linear do consumo de pasto foi compensada pelo teor crescente dos ácidos $\alpha$-linolênico, linoleico e oleico com a quantidade suplementada do GST no concentrado.

Não houve efeito $(\mathrm{P}>0,05)$ sobre a concentração plasmática de glicose, porém a concentração de NEFA aumentou $(\mathrm{P}=0,04)$ com a suplementação crescente de GST (Tab. 4). Provavelmente, o aumento das concentrações de NEFA é explicado pela menor liberação do óleo do GST no rúmen e aumento da absorção dos AGPI no intestino. O aumento da concentração plasmática de NEFA foi explicado por Grummer e Carroll (1991) por meio da maior partição dos AG hidrolizados para glândula mamária comparada aos tecidos, após a suplementação lipídica. Porém, tratou-se de animais no pós-parto imediato. Os resultados do presente estudo corroboram aqueles de DePeters et al. (1989) e Ruegseguer e Schultz (1985). 
Desempenho, composição do leite...

Tabela 4. Concentração plasmática de glicose e de ácidos graxos não-esterificados (NEFA) de vacas em lactação manejadas em pastagem de Brachiaria brizantha cv. Marandu suplementada com 0; 1,3; 2,6 e $3,9 \mathrm{~kg} / \mathrm{vaca} /$ dia de grão de soja tostado no concentrado

\begin{tabular}{lcccccc}
\multirow{2}{*}{ Variável } & \multicolumn{4}{c}{ Concentrado } & \multirow{2}{*}{ EPM } & \multirow{2}{*}{ Equação de regressão } \\
\cline { 2 - 4 } & T0 & T1,3 & T2,6 & T3,9 & & \\
\hline Glicose, mg/dL & 56,0 & 55,6 & 56,8 & 56,8 & 1,21 & ns \\
NEFA, mmol/L & 0,22 & 0,24 & 0,23 & 0,28 & 0,01 & $\hat{y}=0,217+0,014 X\left(\mathrm{r}^{2}=0,05\right)$ \\
\hline
\end{tabular}

Concentrados: T0; T1,3; T2,6 e T3,9 - suplementos concentrados sem inclusão de grão de soja tostado (GST) e com inclusão de 1,3; 2,6 e 3,9kg de GST (base matéria natural); EPM = Erro-padrão da média ; ns = não-significativo $(\mathrm{P}>0,05)$.

Não houve efeito $(\mathrm{P}>0,05)$ dos suplementos na produção e composição do leite, exceto para o teor $(\mathrm{P}=0,10)$ e produção $(\mathrm{P}=0,08)$ de gordura no leite (Tab. 5). A variação do teor e produção de gordura foi de 8 e $9 \%$, respectivamente, para as vacas do T0 para T3,9. Knapp et al. (1991), encontraram aumento do teor e produção de gordura de 3,1 e $3,5 \%$, respectivamente, no leite de vacas que receberam GST na dieta comparado ao daquelas que não receberam GST na dieta. Aumentos semelhantes aos do presente estudo foram encontrados por Faldet et al. (1991) e Ipharraguerre et al. (2005), respectivamente, de 12 e $9 \%$ para a produção de gordura do leite de vacas que também receberam a inclusão de GST na dieta comparado ao das vacas que não receberam o GST.

Os trabalhos com suplementação contendo AGPI relataram efeito negativo desses lipídeos sobre a concentração de gordura do leite, o que comumente é atribuído ao impacto negativo destes sobre a digestibilidade da fibra ou ao aumento da produção de determinados AG trans no rúmen que comprometem a síntese de novo de AG na glândula mamária (Bauman e Grinari, 2003). Provavelmente, no presente estudo, o consumo de pasto propiciou ambiente ruminal adequado para bio-hidrogenação completa, não havendo escape de AG trans suficientes para deprimir a síntese de gordura.

Tabela 5. Produção e composição individual diária do leite de vacas em pastagem de Brachiaria brizantha cv. Marandu suplementada com 0; 1,3; 2,6 e 3,9kg/vaca/dia de grão de soja tostado (GST) no concentrado

\begin{tabular}{|c|c|c|c|c|c|c|}
\hline \multirow{2}{*}{ Variável } & \multicolumn{4}{|c|}{ Concentrado } & \multirow{2}{*}{ EPM } & \multirow{2}{*}{ Equação de Regressão } \\
\hline & T0 & $\mathrm{T} 1,3$ & $\mathrm{~T} 2,6$ & $\mathrm{~T} 3,9$ & & \\
\hline Produção de leite, $\mathrm{kg}$ & 19,9 & 20,4 & 20,2 & 20,4 & 0,259 & ns \\
\hline PLC3,5\%, kg & 20,0 & 21,1 & 21,2 & 21,3 & 0,387 & ns \\
\hline PLC $4,0 \%, \mathrm{~kg}$ & 18,5 & 18,5 & 19,6 & 19,7 & 0,357 & ns \\
\hline Gordura, \% & 3,56 & 3,74 & 3,84 & 3,81 & 0,063 & $\hat{y}=3,60+0,07 X\left(r^{2}=0,02\right)$ \\
\hline Gordura, g & 700,1 & 753,4 & 764,6 & 768,4 & 18,24 & $\hat{y}=714+16,6 X\left(r^{2}=0,03\right)$ \\
\hline Proteína, \% & 2,90 & 2,87 & 2,88 & 2,86 & 0,016 & ns \\
\hline Proteína, g & 575,9 & 584,0 & 579,7 & 580,8 & 8,761 & ns \\
\hline Lactose, $\%$ & 4,34 & 4,34 & 4,32 & 4,34 & 0,019 & ns \\
\hline Lactose, $\mathrm{g}$ & 865,3 & 886,1 & 872,3 & 884,4 & 12,09 & ns \\
\hline Sólidos totais, \% & 11,7 & 11,9 & 12,0 & 12,0 & 0,075 & ns \\
\hline Sólidos totais, $\mathrm{g}$ & 2.332 & 2.420 & 2.410 & 2.430 & 38,40 & ns \\
\hline $\mathrm{ESD}, \%$ & 8,19 & 8,18 & 8,16 & 8,16 & 0,032 & ns \\
\hline $\mathrm{ESD}, \mathrm{kg}$ & 1.631 & 1.666 & 1.646 & 1.662 & 22,94 & ns \\
\hline Teor de NUL, mg/dL & 17,5 & 17,6 & 18,7 & 18,7 & 0,319 & ns \\
\hline Escore Linear CCS & 4,24 & 4,71 & 4,67 & 4,38 & 0,181 & ns \\
\hline
\end{tabular}

Concentrados: T0; T1,3; T2,6 e T3,9 - suplementos concentrados sem inclusão de grão de soja tostado (GST) e com inclusão de 1,3; 2,6 e 3,9kg de GST (base matéria natural); EPM = erro-padrão da média ; ns = não-significativo (P>0,05).PLC3,5\% = produção de leite corrigida para 3,5\% de gordura (Sklan et al., 1992); PLC4,0\% = produção de leite corrigida para 4,0\% de gordura (Nutrient..., 2001); EST = extrato seco desengordurado; NUL = nitrogênio ureico do leite; $\mathrm{CCS}=$ Escore linear de células somáticas do leite. 
Os dados da produção de leite demonstraram que as diferenças no consumo, não limitaram o aporte de nutrientes para a glândula mamária. A média de produção neste estudo foi de $20,2 \mathrm{~kg} / \mathrm{vaca} /$ dia e a maior densidade energética das dietas contendo GST pode ter compensado a sua menor ingestão de MS. Corroborando com isto, não houve alteração da produção de lactose, que é o principal componente osmótico do leite, associado à secreção de água e ao volume de leite. Além disso, a lactose é sintetizada a partir da glicose e a concentração desta foi semelhante $(\mathrm{P}>0,05)$ entre os tratamentos.

\section{CONCLUSÕES}

O menor consumo de pasto com a suplementação crescente de grão de soja tostado no concentrado não alterou a produção de leite. $\mathrm{O}$ aumento no teor e na produção de gordura do leite em função do maior aporte de lipídeos na dieta pode apresentar implicações financeiras positivas para o produtor de leite, devido aos programas de bonificação de pagamento por composição do leite por parte dos laticínios brasileiros.

\section{AGRADECIMENTOS}

À Fundação de Amparo à Pesquisa do Estado de Minas Gerais - FAPEMIG, pelo apoio financeiro ao trabalho (Processo CVZ 1815-06).

\section{REFERÊNCIAS}

ALLEN, M.S. Effects of diet on short-term regulation of feed intake by lactating dairy cattle. J. Dairy Sci., v.83, p.1598-1624, 2000.

BAUMAN, D.E.; GRIINARI, J.M. Nutritional regulation of milk fat synthesis. Annu. Rev. Nutr., v.23, p.203-227, 2003.

DePETERS, E.J.; TAYLOR, S.J.; BALDWIN, R.L. Effect of dietary fat in isocaloric rations on the nitrogen content of milk from Holstein cows. J. Dairy Sci., v.72, p.2949-2957, 1989.

DHIMAN, T.R.; KOREVAAR, A.C.; SATTER, L.D. Particle size of roasted soybeans and the effect on milk production of dairy cows. J. Dairy Sci., v. 80, p. 17221727, 1997

FALDET, M.A.; SATTER, L.D. Feeding heat-treated full fat soybeans to cows in early lactation. J. Dairy Sci., v.74, 3047-3054, 1991.
GRUMMER, R.R.; CARROLL, D.J. Effects of dietary fat on metabolic disorders and reproductive performance of dairy cattle. J. Anim. Sci., v.68, p.3838-3852, 1991.

IPHARRAGUERRE, R.; CLARK, J.H.; FREEMAN, D.E. Rumen fermentation and intestinal supply of Nutrients in dairy cows fed rumen-protected soy products. J. Dairy Sci., v.88, p.2879-2892, 2005.

JORDAN, E.; KENNY, D.; HAWKINS, M. Effect of refined soy or whole soybeans on intake, methane output, and performance of young bulls. J. Anim. Sci., v.84, p.2418-2425, 2006.

KNAPP, D.M.; GRUMMER, R.R.; DENTINE, M.R. The response of lactating dairy cows to increasing levels of whole roasted soybeans. J. Dairy Sci., v.74, p.2563-2572, 1991

KIMURA, F.; MILLER, V.L. Chromic oxide measurement. Improved determination of chromic oxide in cow feed and faeces. J. Agric. Food Quem., v.111, p.633-635, 1952.

LOPES, F.C.F.; AROEIRA, L.J.M.; RODRIGUEZ, N.M. et al. Efeito da suplementação e do intervalo de pastejo sobre a qualidade da forragem e consumo voluntário de vacas Holandês x Zebu em lactação em pastagem de capim-elefante. Arq. Bras. Med. Vet. Zootec, v.56, p.355-362, 2004.

LOPES, F.C.F. Consumo de forrageiras tropicais por vacas em lactação sob pastejo em sistemas de intensivos de produção de leite. Cad. Tec.Vet. Zootec., n. 57 , p.67-117, 2008

LOPES, F.C.F.; RIBEIRO, C.G.S.; RIBEIRO, M.T. et al. Milk fatty acid profile from dairy cows fed increasing levels of soybean oil in diets based on tropical forage. In: INTERNATIONAL SYMPOSIUM ON RUMINANT PHYSIOLOGY. 11., 2009, Clermont-Ferrant. Proceedings... Wageningen: Wageningen Academic Publishers, 2009, p.588-589.

LOPES, F.C.F.; PACIULLO, D.S.C.; MOTTA, E.F. et al. Composição química e digestibilidade ruminal in situ da forragem de quatro espécies do gênero Brachiaria. Arq. Bras. Med. Vet. Zootec., v.62, p.883888, 2010.

McMENIMAN, N.P. Methods of estimating intake of grazing animals. In: REUNIÃO ANUAL SOCIEDADE BRASILEIRA DE ZOOTECNIA, 34., 1997, Juiz de Fora. Anais... Juiz de Fora: SBZ, 1997. p.131-168.

MINSON, D.J. Forage in ruminant nutrition. London:Academic, 1990. 483 p.

NUTRIENT requeriments of dairy cattle, 7.ed. Washington, DC:National Academy, 2001. 381p. 
OFFICIAL methods of analysis. 15.ed., Arlington, VA: AOAC, 1990. 1141p.

OLIVEIRA, R.L.; ASSUNÇÃO, D.M.P.; FREITAS, M.A.A. Efeito do fornecimento de diferentes fontes de lipídios na dieta sobre o consumo, a digestibilidade e o N-ureico plasmático de novilhos bubalinos em confinamento. Rev. Bras. Zootec., v.36, p.733-738, 2007.

PEREIRA, M.N.; ARMENTANO, L.E. Partial replacement of forage with nonforage fiber sources in lactating cow diets. II. Digestion and rumen function. J. Dairy Sci., v.83, p.2876-2875, 2000.

REIS, R.B.; SOUZA, B.M.; Suplementação de vacas leiteiras de vacas em pastagem manejadas intensivamente. In: Simpósio sobre Bovinocultura leiteira, 6.,2007. Piracicaba. Anais...Piracicaba: Esalq/USP, 2007. p.151-182.

RUEGSEGGER, G.; SCHULTZ, L.H. Response of high producing dairy cows in early lactation to the feeding of heat-treated whole soybeans. J. Dairy Sci., v.68, p.3272-3279, 1985.

SANTOS, F.A.P.; SANTOS, J.E.P.; THEURER, C.B. Effects of rumen-undegradable protein on dairy cow performance: a 12-year literature review. J. Dairy Sci.,v.81, p.3182-3213, 1998.

SILVA, D.J.; QUEIROZ, A.C. Análise de alimentos.: métodos químicos e biológicos. 3. ed. Viçosa: UFV, 2002. 235p.

SILVA, L.D.F.; RAMOS, B.M.O.; RIBEIRO, E.L.A. et al. Degradabilidade ruminal in situ da matéria seca e proteína bruta de duas variedades de grão de soja com diferentes teores de inibidor de tripsina, em bovinos. Rev. Bras. Zootec., v.31, p.1251-1257, 2002.

SILVA, M.M.C.; RODRIGUES, M.T.; RODRIGUES, C.A.F. et al. Efeito da suplementação de lipídios sobre a digestibilidade e os parâmetros da fermentação ruminal em cabras leiteiras. Rev. Bras. Zootec., v.36, p.246-256, 2007.

SKLAN, D.; ASHKENAZI, R.; BRAUN, A. et al. Fatty acids, calcium soaps of fatty acids and cottonseeds fed to high yielding cows. J. Dairy Sci., v.75, v.2463-2472, 1992.
SOUZA, A.R.D.L.; MEDEIROS, S.R.; MORAIS, M.G. et al. Dieta com alto teor de gordura e desempenho de tourinhos de grupos genéticos diferentes em confinamento. Pesq. Agropec. Bras., v.44, p. 746-753, 2009.

STATISTICAL analyses systems - SAS: User's guide: Statistics, Version 5.ed. Cary, NC: SAS Institute Inc., 2002.

THOMPSON, G.E.; CHRISTIE, W.W. Extration of plasma triacylglycerols by the mammary gland of the lactating cow. J. Dairy Res., v.58, p.251-255, 1991.

TILLEY, J.M.A.; TERRY, R.A. A two-stage technique for the in vitro digestion of forage crops. $J$. Br. Grassl. Soc., v.18, p.104-111, 1963.

TRICON, S.; YAQOOB, P. Conjugated linoleic acid and human health: a critical evaluation of the evidence. Curr. Opin. Clin. Nut. Metab. Care, v.9, p.105-110, 2006.

VALADARES FILHO, S.C., MACHADO, P.A.S.; CHIZZOTTI, M.L. et al. Tabelas brasileiras de composição de alimentos para bovinos, 3.ed. Viçosa:UFV, DZOO, 2010. 502p.

VAN SOEST, P.J. Nutrition ecology of the ruminant. 2.ed. Ithaca: Cornell University Press, 1994. 476p.

VARGAS, L.H.; LANA, R.P.; JHAM, G.N. et al. Adição de Lipídios na ração de vacas leiteiras: parâmetros fermentativos ruminais, produção e composição do leite. Rev. Bras. Zootec., v.31, supl., p.522-529, 2002.

WILLIANS, C.H.; DAVID, D.J.; ILSMAA, O. The determination of chromic oxide in falces samples by atomic absorption spectrophotometry. J. Agric. Sci., v.59, p.381-385, 1962.

WONSIL, B.J.; HERBEIN, J.H.; WATKINS, B.A. Dietary and ruminally derived trans-18:1 fatty acids alter bovine milk lipids. J. Nutr., v.124, p.556-565, 1994. 